\title{
Advanced Materials for Applications in Acoustics and Vibration
}

\author{
Yehia A. Bahei-El-Din ${ }^{1}$ and Mohammad Tawfik ${ }^{2}$ \\ ${ }^{1}$ Centre for Advanced Materials, The British University in Egypt, El-Shorouk City 11837, Cairo, Egypt \\ ${ }^{2}$ Noon for Research and Development, Cairo, Egypt \\ Correspondence should be addressed to Yehia A. Bahei-El-Din, ybahei@bue.edu.eg
}

Received 8 November 2009; Accepted 8 November 2009

Copyright (c) 2009 Y. A. Bahei-El-Din and M. Tawfik. This is an open access article distributed under the Creative Commons Attribution License, which permits unrestricted use, distribution, and reproduction in any medium, provided the original work is properly cited.

Research in advanced materials is progressing on many fronts with significant developments in composite, smart and nano-scale materials. Industrial applications have also benefited from advances in material science and engineering. Substantial work, however, lies ahead to integrate advanced materials in engineering applications. One such application which has significant impact on both the economy and quality of life is acoustics and vibrations of materials and mechanical systems. Generally, however, materials and dynamics are considered by different groups with little or no interaction. The purpose of the International Conference on Advanced Materials for Application in Acoustics and Vibration (AMAAV) - held in Cairo, Egypt from January 4 to 6, 2009-was to bridge the gap between research and application in the significant field of dynamics, with emphasis on materials, by bringing together regional and international researchers and engineers to present and discuss recent developments in utilizing advanced materials in applications related to acoustics and vibration. It provided a forum for disseminating the latest research findings in material development, modeling, and testing under service loads and environments, derived from real life applications in which acoustics and vibrations play a major role in their design and performance.

This special issue of Advances in Acoustics and Vibration contains papers contributed to AMAAV conference. The contributions contained in this issue address both purely dynamic problems and dynamics of/advanced materials. In the latter category, Buonsanti et al. investigate detection of defects in carbon/epoxy composites using ultrasonic testing, and Eldalil and Baz study active control of cylindrical shells subjected to internal pressure pulse using piezoelectric materials. Vibration problems are investigated in four contributed papers; Buonsanti et al. considered the effect of railway-induced vibrations on buildings; Di Mino et al. also considered vibrations caused by railways and investigated the role of open trenches in their reduction; Eldalil and Baz studied the effect of periodic stiffeners on the dynamic stability of cylindrical shells subjected to internal pressure pulse; Patwari et al. presented a method for dynamic characterization of vertical machining. In acoustics, Ivansson considered the design of acoustic frequency insulators using phononic crystals.

The time and effort spent by the authors in participating in the meeting and preparing the manuscripts for this special issue is greatly appreciated.

Yehia A. Bahei-El-Din Mohammad Tawfik 

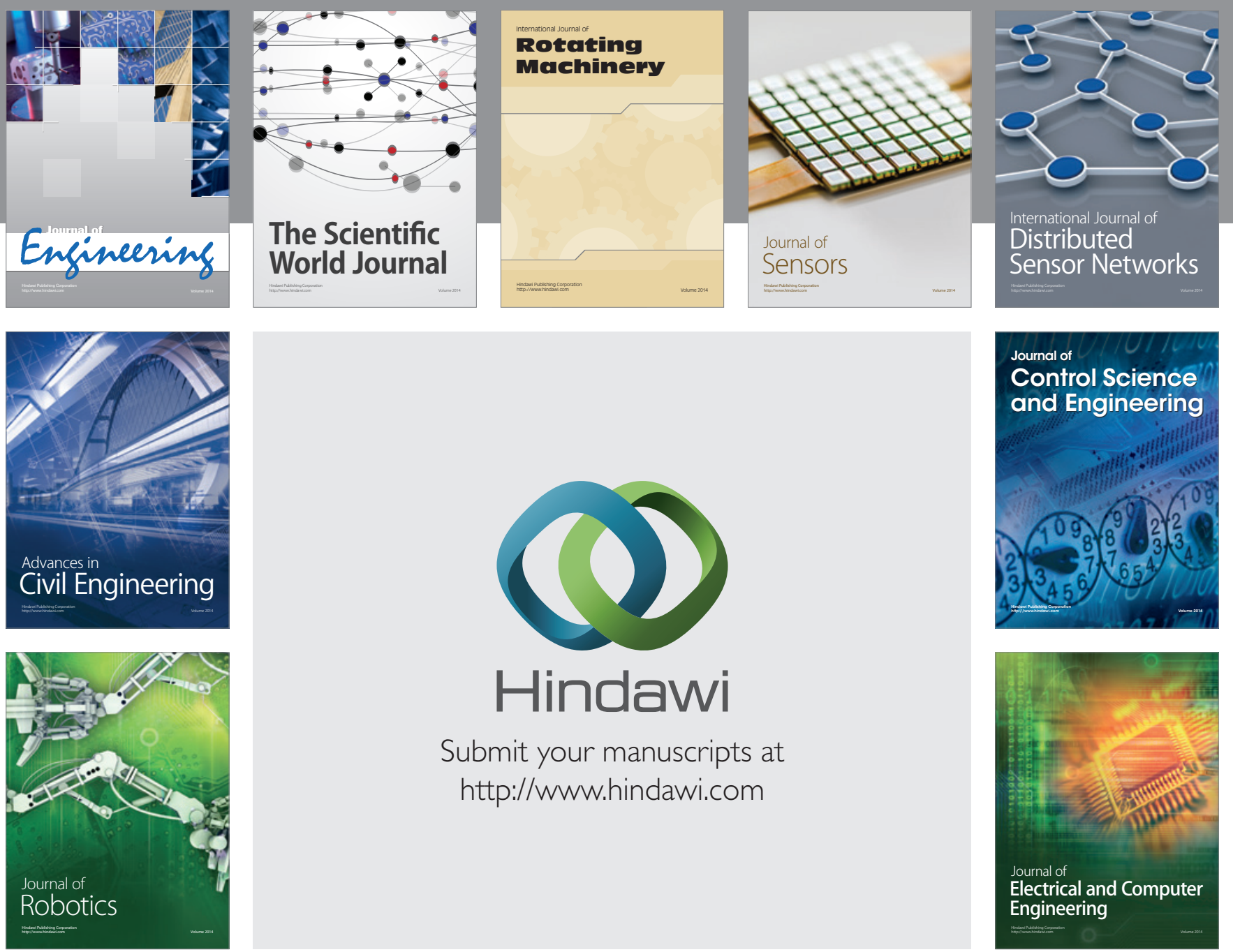

Submit your manuscripts at

http://www.hindawi.com
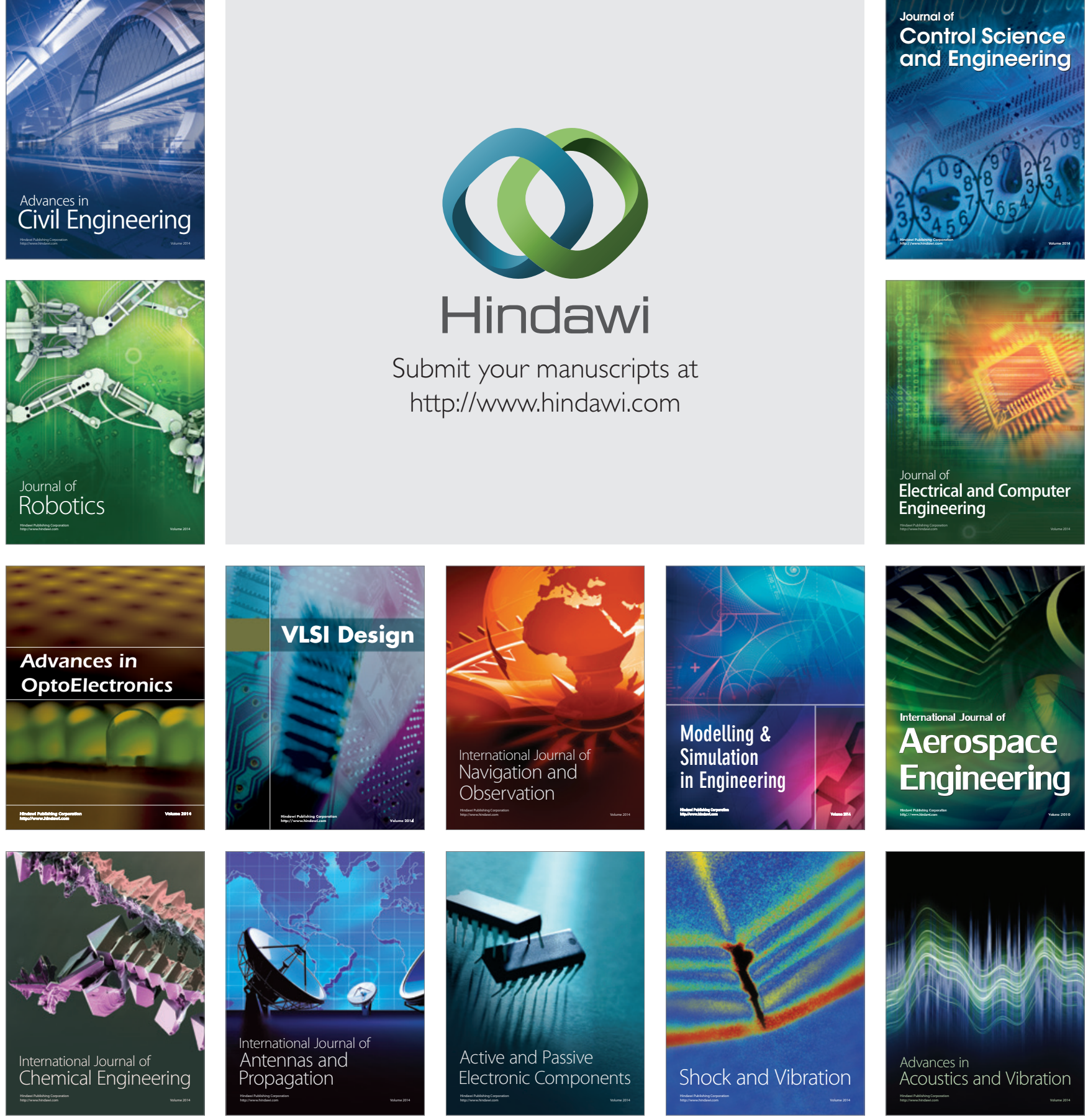